\title{
Preoperative Localization and Intraoperative Parathyroid Hormone Assay in Korean Patients with Primary Hyperparathyroidism
}

\author{
Eirie Cho ${ }^{1}$, Jung Mi Chang ${ }^{1}$, Seok Young Yoon ${ }^{1}$, Gil Tae Lee ${ }^{1}$, Yun Hyi Ku ${ }^{1}$, Hong Il Kim ${ }^{1}$, Myung-Chul Lee ${ }^{2}$, \\ Guk Haeng Lee ${ }^{2}$, Min Joo Kim ${ }^{1}$
}

Departments of ${ }^{1}$ Internal Medicine and ${ }^{2}$ Otorhinolaryngology, Korea Cancer Center Hospital, Seoul, Korea

Background: The intraoperative parathyroid hormone (IOPTH) assay is widely used in patients with primary hyperparathyroidism (PHPT). We investigated the usefulness of the IOPTH assay in Korean patients with PHPT.

Methods: We retrospectively reviewed the data of 33 patients with PHPT who underwent parathyroidectomy. Neck ultrasonography (US) and ${ }^{99 \mathrm{~m}} \mathrm{Tc}$-sestamibi scintigraphy (MIBI scan) were performed preoperatively and IOPTH assays were conducted.

Results: The sensitivity of neck US and MIBI scans were $91 \%$ and $94 \%$, respectively. A 50\% decrease in parathyroid hormone (PTH) levels 10 minutes after excision of the parathyroid gland was obtained in 91\% (30/33) of patients and operative success was achieved in $97 \%$ (32/33) of patients. The IOPTH assay was $91 \%$ true-positive, $3 \%$ true-negative, $0 \%$ false-positive, and $6 \%$ falsenegative. The overall accuracy of the IOPTH assay was $94 \%$. In five cases with discordant neck US and MIBI scan results, a sufficient decrease in IOPTH levels helped the surgeon confirm the complete excision of the parathyroid gland with no additional neck exploration.

Conclusion: The IOPTH assay is an accurate tool for localizing hyperfunctioning parathyroid glands and is helpful for evaluating cases with discordant neck US and MIBI scan results.

Keywords: Parathyroid hormone; Parathyroid neoplasms; Hyperparathyroidism, primary; Technetium Tc 99m sestamibi; Ultrasonography

\section{INTRODUCTION}

Primary hyperparathyroidism (PHPT) is common and the incidence of PHPT has increased since the addition of serum calcium levels to the standard chemistry panel [1]. The estimated incidence was approximately 22 per 100,000 people in the United States [1]. The only definite therapy for PHPT is surgery, which is recommended for symptomatic and some as-

Received: 8 January 2014, Revised: 15 April 2014,

Accepted: 26 April 2014

Corresponding author: Min Joo Kim

Department of Internal Medicine, Korea Cancer Center Hospital, 75 Nowon-ro, Nowon-gu, Seoul 139-706, Korea

Tel: +82-2-970-1215, Fax: +82-2-970-2438, E-mail: chorong24@gmail.com ymptomatic patients [2]. The goal of surgery is to remove the abnormal hyperfunctioning parathyroid glands, preserving the normal parathyroid glands to achieve eucalcemia. Therefore, it is important to localize the abnormal glands for a successful surgery. Most cases ( $80 \%$ to $85 \%$ ) of PHPT are caused by a single abnormal gland while a minority of cases $(10 \%$ to $15 \%)$ are due to multiple abnormal glands [3]. In addition, some parathyroid glands ( $5 \%$ to $20 \%$ ) are found in ectopic locations.

Copyright $\odot 2014$ Korean Endocrine Society

This is an Open Access article distributed under the terms of the Creative Commons Attribution Non-Commercial License (http://creativecommons.org/ licenses/by-nc/3.0/) which permits unrestricted non-commercial use, distribution, and reproduction in any medium, provided the original work is properly cited. 
Before recent advances in imaging technology, bilateral neck exploration for all four parathyroid glands was the gold standard in patients with PHPT. Several imaging methods, such as neck ultrasonography (US) and ${ }^{99 \mathrm{~m}}$ Tc-setamibi scintigraphy (MIBI scan) have been developed to localize abnormal parathyroid glands. Consequently, neck US and MIBI scans have allowed for minimally invasive parathyroidectomy [4]. The sensitivities of neck US and MIBI scans are reported to be $65 \%$ to $74 \%$ and $68 \%$ to $80 \%$, respectively $[5,6]$.

The intraoperative parathyroid hormone (IOPTH) assay is another important tool for minimally invasive parathyroidectomy [4]. The IOPTH assay, first proposed by Nussbaum et al. [7] in 1988, became commercially available in 1996. Since parathyroid hormone (PTH) has a half-life of 2 to 3 minutes, its level is reduced significantly by 5 to 10 minutes after the removal of the abnormal glands. The IOPTH assay helps to confirm the identification and removal of all hyperfunctioning parathyroid glands. Consequently, the IOPTH assay helps the surgeon decide whether to complete the operation or do further neck exploration.

It is well established that the IOPTH assay can improve the cure rate in patients with PHPT [8,9]. However, there is a lack of data regarding this procedure in Korea $[10,11]$. Therefore, we investigated the usefulness of the IOPTH assay in Korean patients with PHPT.

\section{METHODS}

We retrospectively reviewed patients with PHPT who underwent surgery at the Korea Cancer Center Hospital between December 2008 and April 2013. PHPT was defined as hypercalcemia with inappropriately elevated PTH level compared with the normal reference range. This study was approved by the Institutional Protocol Review Board of the Korea Cancer Center Hospital. A total of 36 patients underwent surgery, but we excluded three patients for whom IOPTH was not evaluated. All remaining 33 patients were included in this study.

Calcium, albumin, and phosphorous were measured by using a Hitachi Modular P800 Chemistry Analyzer (Hitachi, Tokyo, Japan). The reference ranges for calcium and phosphorus were 8.6 to $10.2 \mathrm{mg} / \mathrm{dL}$ and 2.7 to $4.5 \mathrm{mg} / \mathrm{dL}$, respectively. The corrected calcium level was calculated as follows: calcium $(\mathrm{mg} / \mathrm{dL})+[4-$ albumin $(\mathrm{g} / \mathrm{dL})]$.

The neck US was performed with the use of a high resolution 5 to $13 \mathrm{MHz}$ transducer and HI VISION Ascendus ultrasound system (Hitachi Aloka Medical). An oval-shaped and hypoechoic mass compared to the overlying thyroid gland was usually considered to be a parathyroid mass (positive imaging). For the MIBI scan, ${ }^{99 \mathrm{~m}} \mathrm{Tc}$ sestamibi was administrated intravenously and images at 5 to 10 minutes (early) and at 3 hours (delayed) were obtained. Positive scan imaging was defined as having foci of retained radiotracer in the delayed image. We reviewed the results of neck US and MIBI scans, which contained the information for the locations and numbers of abnormal parathyroid masses. Among the 33 patients, preoperative neck US and MIBI scans were conducted for 32 patients.

Most patients underwent minimally invasive parathyroidectomies except for particular cases, such as concomitant thyroid disease or multiple endocrine neoplasia (MEN). The surgery was performed through a minimal transverse incision (i.e., $<2.5 \mathrm{~cm}$ ) directly over the localized parathyroid gland. The remaining parathyroid glands were not electively explored.

In the IOPTH assay, a "preoperative" PTH level was defined as the highest PTH level measured before the operation. A "pre-excision" PTH level was measured after the artery to the suspected abnormal parathyroid gland was ligated in some patients $(n=20)$. A postoperative PTH level was measured from blood drawn at 5, 10, and 15 minutes after removal of the suspected abnormal parathyroid glands. All blood samples during the operation were obtained from an intravenous catheter in the foot. For PTH testing, blood samples were collected in a plain bottle, centrifuged for 10 minutes, and then placed in the autoanalyzer (the ADVIA Centaur immunoassay, Siemens Health Diagnostics, Inc., Tarrytown, NY, USA). The reference range for PTH was 14 to $72 \mathrm{pg} / \mathrm{mL}$.

Operative success was defined as eucalcemia at 6 months after parathyroidectomy. Electronic medical records were used to obtain postsurgical information, such as serum calcium and PTH levels, and histories of taking medicine (i.e., calcium supplements).

\section{Statistical analysis}

Data were presented as mean $\pm \mathrm{SD}$. The sensitivity, positive predictive value (PPV), and overall accuracy of both neck US and MIBI scans were calculated. The efficacy of IOPTH for operative success was expressed as overall accuracy.

\section{RESULTS}

\section{Characteristics of the study population}

A total of 33 patients with PHPT who underwent parathyroidectomies were included in this analysis. The mean age at time 
of surgery was $51 \pm 12$ years and $72 \%$ of the patients were female (Table 1). Most patients had sporadic PHPT and only two patients had familial PHPT (MEN type 1).

One gland was removed in most patients, two glands in one patient, and four glands in the two patients who were diagnosed with MEN. In cases where four hyperfunctioning glands were present, total parathyroidectomy was performed with autotransplantation in the forearm. Surgical pathology reports showed that there were $26(79 \%)$ parathyroid adenomas, three $(10 \%)$ parathyroid carcinomas, three $(10 \%)$ parathyroid hyperplasias, and one (1\%) normal parathyroid gland. Among the 29 patients with parathyroid adenomas or carcinomas, 28 patients had a single adenoma or carcinoma and one patient had two adenomas.

\section{Neck US and MIBI scan}

In preoperative imaging studies, the results of neck US and

\begin{tabular}{lc}
\multicolumn{2}{l}{ Table 1. Baseline Characteristics of the 33 Study Patients } \\
\hline Characteristic & Value \\
\hline Age at operation, yr & $51 \pm 12$ \\
Sex, male/female & $9 / 24$ \\
Preoperative PTH, pg/mL & $378 \pm 423$ \\
Preoperative Ca, $\mathrm{mg} / \mathrm{dL}$ & $11.1 \pm 0.8$ \\
Preoperative corrected Ca, $\mathrm{mg} / \mathrm{dL}$ & $10.6 \pm 1.0$ \\
Preoperative $\mathrm{P}, \mathrm{mg} / \mathrm{dL}$ & $2.7 \pm 0.6$ \\
Postoperative Ca, $\mathrm{mg} / \mathrm{dL}$ & $9.1 \pm 1.0$ \\
Postoperative corrected Ca, $\mathrm{mg} / \mathrm{dL}$ & $8.7 \pm 0.8$ \\
Postoperative P, $\mathrm{mg} / \mathrm{dL}$ & $3.4 \pm 0.7$ \\
Postoperative PTH, pg/mL & $36.1 \pm 31.5$ \\
\hline Values are expressed as mean $\pm \mathrm{SD}$. & \\
PTH, parathyroid hormone.
\end{tabular}

Table 2. Accuracies of Neck US and MIBI Scan: Agreement between Results of Neck US or MIBI Scan and Results of Surgical Pathology

\begin{tabular}{lcccccc}
\hline \multirow{2}{*}{ Variable } & \multicolumn{2}{c}{ Neck US } & & \multicolumn{2}{c}{ MIBI scan } \\
\cline { 2 - 3 } \cline { 5 - 6 } Agree & Positive & Negative & & Positive & Negative \\
\hline Disagree & 29 & 0 & & 29 & 0 \\
Sensitivity & 0 & & 3 & & 2 & 2 \\
PPV & & 90.6 & & & 93.5 \\
Accuracy & & 100 & & \multicolumn{2}{c}{93.5} \\
\hline
\end{tabular}

US, ultrasonography; MIBI scan, ${ }^{99 \mathrm{~m}} \mathrm{Tc}$-setamibi scintigraphy; PPV, positive predictive value.
MIBI scans were positive in 29 (91\%) and 31 (94\%) patients, respectively. All positive results from neck US corresponded with those of surgical pathology, but two positive results from MIBI scans did not. A single abnormal gland was suspected by performing neck US in $26(81 \%)$ patients and in $28(85 \%)$ patients by MIBI scans. When the locations and numbers of abnormal parathyroid masses on the neck US were compared with those on the MIBI scan, the neck US and MIBI findings were discordant in five cases. In those cases, the results of the neck US were correct in three cases and the results of MIBI scans were correct in two cases. The sensitivities of neck US and MIBI scans were 91\% and 94\%, respectively (Table 2). The PPVs were $100 \%$ for neck US and 94\% for MIBI scans. The accuracies of neck US and MIBI scans were estimated as $91 \%$ and $88 \%$, respectively.

\section{IOPTH assay}

The mean preoperative PTH level was $378 \pm 423$ pg $/ \mathrm{mL}$. Postoperative PTH levels at 5, 10, and 15 minutes after excision decreased by $69 \%, 73 \%$, and $78 \%$, respectively, compared to preoperative PTH levels (Fig. 1). A 50\% decrease in PTH levels 10 minutes after excision is an accepted criteria to confirm the complete removal of hyperfunctioning parathyroid glands [12]; $91 \%(30 / 33)$ of patients in our study met this criteria. Only three patients in our study did not meet the criteria. The pre-excision PTH levels were obtained from 20 patients. Among these 20 patients, the mean pre-excision PTH level was $331 \pm$ $329 \mathrm{pg} / \mathrm{mL}$. Postoperative PTH levels at 5, 10, and 15 minutes after excision decreased by $38 \%, 51 \%$, and $56 \%$, respectively, compared to pre-excision PTH levels (Fig. 1). Only 60\% (12/

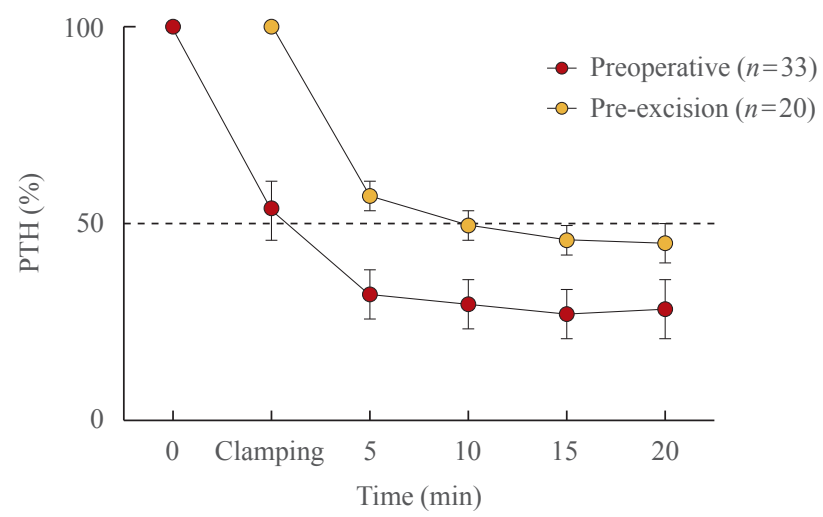

Fig. 1. Intraoperative parathyroid hormone assay. Percent change in parathyroid hormone (PTH) levels when using preoperative PTH levels as a baseline (shown as red circle) and when using preexcision PTH levels as a baseline (shown as yellow circle). 
20) of patients achieved a 50\% decrease in PTH levels 10 minutes after excision and $40 \%(8 / 20)$ of patients did not.

Six months following parathyroidectomy, operative success was achieved in 97\% (32/33) of patients. Half of the patients (17/32) took calcium supplementation and the remaining patients had eucalcemia without calcium supplementation. However, only one patient had hypercalcemia and an increased PTH level following parathyroidectomy. This patient was one out of three patients whose intraoperative PTH level at 10 minutes did not meet the criteria. The IOPTH assay was $91 \%$ true-positive, $3 \%$ true-negative, $0 \%$ false-positive, and $6 \%$ false-negative, making the overall accuracy of the IOPTH assay $94 \%$. However, when using pre-excision PTH levels as a baseline, the accuracy of the IOPTH assay decreased to $65 \%$. When using the highest of either preoperative or pre-excision PTH level as baseline, the accuracy of the IOPTH assay did not change (94\%).

In the five cases with discordant neck US and MIBI scan results the surgeon identified suspicious parathyroid masses, confirmed the complete removal with IOPTH assay, and did not perform further neck exploration.

\section{DISCUSSION}

A total of 33 patients underwent parathyroidectomies and all of them, except for one patient, were cured. The accuracies of neck US, MIBI scans, and the IOPTH assay were 91\%, 88\%, and $94 \%$, respectively.

Minimally invasive parathyroidectomy is preferred because it reduces operative time, length of hospital stay, and risk of complications such as hypocalcemia and nerve injury $[13,14]$. Preoperative localization of abnormal hyperfunctioning parathyroid glands is mandatory for minimally invasive parathyroidectomy. Neck US and MIBI scans have been commonly used for preoperative localization of abnormal glands [4]. In this study, both neck US and MIBI scans showed high sensitivity ( $91 \%$ for neck US and $94 \%$ for MIBI scans). The sensitivity measured in this study was slightly higher than those of previous studies $[5,6]$. A possible reason for this difference is that neck US and MIBI scans can detect a single adenoma more accurately than multiple adenomas or hyperplasia $[15,16]$, and most patients in this study had a single adenoma. In one study, the sensitivities of neck US and MIBI scans decreased from $74 \%$ and $80 \%$ in single-gland disease to $22 \%$ and $22 \%$ in multiple-gland disease, respectively [17]. In addition, the experience of the radiologist for neck US might influ- ence the sensitivity of neck US. It is already known that both US and MIBI scans can predict the presence and location of parathyroid adenomas more accurately than neck US or MIBI scans alone [6,18]. In this study, the sensitivity of combined neck US and MIBI scans was $100 \%$. However, there was some discrepancy in the neck US and MIBI scan results of 5 patients.

The accuracy of the IOPTH assay in this study was $94 \%$, which is similar to the rates determined in previous studies $[19,20]$. The accuracy of IOPTH assays is dependent on sample timing and the criteria for predicting operative success. There are several interpretation criteria for IOPTH assays such as the Miami, Vienna, Rome, and Halle criteria [12,21,22]. Because the Rome and Halle criteria showed higher false negative results than did other criteria, those seem to be inappropriate for use with a single parathyroid adenoma $[21,23]$. In both Miami and Vienna criteria, a $50 \%$ or more decrease in PTH levels from baseline at 10 minutes after excision predicts operative success. The Miami criteria uses the highest pre-incision or pre-excision PTH levels [12] and the Vienna criteria uses the pre-incision PTH level as a baseline [21]. In this study, subgroup analysis showed that using pre-excision PTH levels as a baseline decreased the accuracy of the IOPTH assay from $94 \%$ to $65 \%$ and using the highest pre-incision or pre-excision PTH levels as a baseline did not increase the accuracy of the IOPTH assay. Previous studies reported that the Miami criteria slightly reduced false-negative results and increased false-positive results compared to the Vienna criteria $[12,21,23]$. Therefore, in order not to miss additional parathyroid adenomas and prevent re-operation, the Vienna criteria may be more beneficial than the Miami criteria.

IOPTH assays can improve the success rate of surgery $[8,9]$. A sufficient decrease in the IOPTH level can help the surgeon confirm when a complete and successful excision of abnormal parathyroid gland has been achieved. In this study, the IOPTH assay was especially useful in the five cases that showed discordance in neck US and MIBI scan results. In those cases, the surgeon confirmed the complete excision and did not perform further neck exploration because there was a more than 50\% decrease in PTH levels 10 minutes after parathyroid gland excision. In contrast, an insufficient decrease in IOPTH levels indicates the presence of an additional abnormal parathyroid gland and the need for subsequent neck exploration. However, the IOPTH assay is limited by false-positive and false-negative results. In particular, false-negative results from IOPTH assays can lead to unnecessary exploration, resulting in an in- 
crease of cost and morbidity related to surgery [24]. The falsenegative rate has been reported to range from $0.9 \%$ to $9 \%$ in previous studies $[12,19,24,25]$, and it was $6 \%(2 / 33)$ in this study. Two false-negative cases had a single parathyroid adenoma and the results from neck US and MIBI scans were concordant. Therefore, even if the IOPTH assay shows a negative result, the possibility of a false-negative result should be considered if the findings from neck US and MIBI scans are concordant in cases of a single adenoma.

This study has some limitations. First, blood samples for measuring preoperative PTH levels were obtained the day before the operation in this study, whereas in other studies, blood was collected in the operating room before the skin incision was made (preincision). Considering the importance of sampling time, the absence of pre-incision samples is a limitation in our study. Second, one-third of patients underwent thyroid surgery at the same time. PHPT was often diagnosed during work-up for thyroid cancer, because of a high volume of thyroid cancer in our hospital.

In conclusion, the IOPTH assay can be used to accurately localize abnormal hyperfunctioning parathyroid glands and help surgeons confirm the complete and successful resection of abnormal glands.

\section{CONFLICTS OF INTEREST}

No potential conflict of interest relevant to this article was reported.

\section{REFERENCES}

1. Wermers RA, Khosla S, Atkinson EJ, Achenbach SJ, Oberg AL, Grant CS, Melton LJ 3rd. Incidence of primary hyperparathyroidism in Rochester, Minnesota, 1993-2001: an update on the changing epidemiology of the disease. J Bone Miner Res 2006;21:171-7.

2. Bilezikian JP, Khan AA, Potts JT Jr; Third International Workshop on the Management of Asymptomatic Primary Hyperthyroidism. Guidelines for the management of asymptomatic primary hyperparathyroidism: summary statement from the third international workshop. J Clin Endocrinol Metab 2009;94:335-9.

3. Marcocci C, Cetani F. Clinical practice. Primary hyperparathyroidism. N Engl J Med 2011;365:2389-97.

4. Lew JI. Current understanding and treatment of primary hyperparathyroidism. Endocrinol Metab 2011;26:109-17.
5. Arici C, Cheah WK, Ituarte PH, Morita E, Lynch TC, Siperstein AE, Duh QY, Clark OH. Can localization studies be used to direct focused parathyroid operations? Surgery 2001; 129:720-9.

6. Siperstein A, Berber E, Mackey R, Alghoul M, Wagner K, Milas M. Prospective evaluation of sestamibi scan, ultrasonography, and rapid PTH to predict the success of limited exploration for sporadic primary hyperparathyroidism. Surgery 2004;136:872-80.

7. Nussbaum SR, Thompson AR, Hutcheson KA, Gaz RD, Wang CA. Intraoperative measurement of parathyroid hormone in the surgical management of hyperparathyroidism. Surgery 1988;104:1121-7.

8. Chen H, Pruhs Z, Starling JR, Mack E. Intraoperative parathyroid hormone testing improves cure rates in patients undergoing minimally invasive parathyroidectomy. Surgery 2005;138:583-7.

9. Barczynski M, Konturek A, Cichon S, Hubalewska-Dydejczyk A, Golkowski F, Huszno B. Intraoperative parathyroid hormone assay improves outcomes of minimally invasive parathyroidectomy mainly in patients with a presumed solitary parathyroid adenoma and missing concordance of preoperative imaging. Clin Endocrinol (Oxf) 2007;66:878-85.

10. Kim WY, Lee JB, Kim HY. Efficacy of intraoperative parathyroid hormone monitoring to predict success of parathyroidectomy for secondary hyperparathyroidism. J Korean Surg Soc 2012;83:1-6.

11. Lee JH, Moon KR, Kim HJ, Chung SM, Kim HS. Usefulness of intraoperative parathyroid hormone assay in minimally invasive surgery. Korean J Otorhinolaryngol-Head Neck Surg 2009;52:426-30.

12. Carneiro DM, Solorzano CC, Nader MC, Ramirez M, Irvin GL 3rd. Comparison of intraoperative iPTH assay (QPTH) criteria in guiding parathyroidectomy: which criterion is the most accurate? Surgery 2003;134:973-9.

13. Norman J, Chheda H, Farrell C. Minimally invasive parathyroidectomy for primary hyperparathyroidism: decreasing operative time and potential complications while improving cosmetic results. Am Surg 1998;64:391-5.

14. Udelsman R. Six hundred fifty-six consecutive explorations for primary hyperparathyroidism. Ann Surg 2002; 235:665-70.

15. Haciyanli M, Lal G, Morita E, Duh QY, Kebebew E, Clark $\mathrm{OH}$. Accuracy of preoperative localization studies and intraoperative parathyroid hormone assay in patients with primary hyperparathyroidism and double adenoma. J Am 
Coll Surg 2003;197:739-46.

16. Sugg SL, Krzywda EA, Demeure MJ, Wilson SD. Detection of multiple gland primary hyperparathyroidism in the era of minimally invasive parathyroidectomy. Surgery 2004; 136:1303-9.

17. Siperstein A, Berber E, Barbosa GF, Tsinberg M, Greene AB, Mitchell J, Milas M. Predicting the success of limited exploration for primary hyperparathyroidism using ultrasound, sestamibi, and intraoperative parathyroid hormone: analysis of 1,158 cases. Ann Surg 2008;248:420-8.

18. Lumachi F, Zucchetta P, Marzola MC, Boccagni P, Angelini F, Bui F, D'Amico DF, Favia G. Advantages of combined technetium-99m-sestamibi scintigraphy and highresolution ultrasonography in parathyroid localization: comparative study in 91 patients with primary hyperparathyroidism. Eur J Endocrinol 2000;143:755-60.

19. Vignali E, Picone A, Materazzi G, Steffe S, Berti P, Cianferotti L, Cetani F, Ambrogini E, Miccoli P, Pinchera A, Marcocci C. A quick intraoperative parathyroid hormone assay in the surgical management of patients with primary hyperparathyroidism: a study of 206 consecutive cases. Eur J Endocrinol 2002;146:783-8.

20. Miura D, Wada N, Arici C, Morita E, Duh QY, Clark OH. Does intraoperative quick parathyroid hormone assay improve the results of parathyroidectomy? World J Surg 2002;
26:926-30.

21. Riss P, Kaczirek K, Heinz G, Bieglmayer C, Niederle B. A "defined baseline" in PTH monitoring increases surgical success in patients with multiple gland disease. Surgery 2007;142:398-404.

22. Lombardi CP, Raffaelli M, Traini E, Di Stasio E, Carrozza C, De Crea C, Zuppi C, Bellantone R. Intraoperative PTH monitoring during parathyroidectomy: the need for stricter criteria to detect multiglandular disease. Langenbecks Arch Surg 2008;393:639-45.

23. Barczynski M, Konturek A, Hubalewska-Dydejczyk A, Cichon S, Nowak W. Evaluation of Halle, Miami, Rome, and Vienna intraoperative iPTH assay criteria in guiding minimally invasive parathyroidectomy. Langenbecks Arch Surg 2009;394:843-9.

24. Stalberg P, Sidhu S, Sywak M, Robinson B, Wilkinson M, Delbridge L. Intraoperative parathyroid hormone measurement during minimally invasive parathyroidectomy: does it "value-add" to decision-making? J Am Coll Surg 2006;203: 1-6.

25. Garner SC, Leight GS Jr. Initial experience with intraoperative PTH determinations in the surgical management of 130 consecutive cases of primary hyperparathyroidism. Surgery 1999;126:1132-7. 